\title{
Empirische Untersuchung von Prioritätsregeln füir die ressourcenbeschränkte Projektplanung mit zeitlichen Minimal- und Maximalabständen
}

\author{
Birger Franck
}

Summary: After the definition of the Resource-Constrained Project Scheduling Problem with minimal and maximal time lags (RCPSP/max) we propose a priority-rule based method for RCPSP/max, which first treats the cycle structures seperately and performs a constraction of the cyclic network to an acyclic network. We use test problems generated by the problem generator ProGen/max for the algorithm evaluation. We specify some of the choosen parameters. Finally we present the results of an empirical analysis which differ slightly from other investigatiions.

\section{Einleitung}

Für die ressourcenbeschränkte Projektplanung mit zeitlichen Minimalabständen und Minimierung des Projektendtermins (RCPSP) gibt es eine Vielzahl exakter und heuristische Verfahren. Bei den exakten Verfahren handelt es sich meistens um Branch-and-Bound-Verfahren, bei den heuristischen um PrioritätsregelVerfahren. Während für das RCPSP vielfältige Untersuchungen angestellt wurden, sind für das RCPSP mit zeitlichen Minimal- und Maximalabständen (RCPSP/max) bisher nur wenige Verfahren entwickelt worden. Ein Branch-andBound-Verfahren findet sich in Bartusch et al. 1988. Prioritätsregelverfahren werden von Zhan 1991 und verkürzte Branch-and-Bound-Verfahren von Brinkmann und Neumann 1995 vorgestellt. Bei den beiden heuristischen Verfahren wird zwischen direkten und Kontraktions-Methoden unterschieden. Im folgenden wollen wir uns nur mit der Kontraktions-Methode beschäftigen, da diese sich als die bessere herausgestellt hat (Brinkmann und Neumann 1995). Es wird ein gegenüber Zhan verbessertes Prioritätsregelverfahren vorgestellt. Anhand von Testproblemen, die mit den neuen Generator ProGen/max (Schwindt 1995) erzeugt wurden, wird ermittelt, welche Prioritätsregeln die besten Ergebnisse liefern.

\section{Das RCPSP/max}

Wir gehen von einem Vorgangsknoten-Netzwerk aus. Alle Vorrangbeziehungen seien Start-zu-Start-Beziehungen. Für zeitlichen Minimalabstände $T_{i j}^{\text {min }} \geq 0$ 
zwischen zwei Vorgängen $i$ und $j$ gelte $S T_{j}-S T_{i} \geq T_{i j}^{m i n}$ und für die zeitlichen Maximalabstände $T_{i j}^{\text {max }} \geq 0$ zwischen zwei Vorgängen $i$ und $j$ gelte $S T_{j}-S T_{i} \leq T_{i j}^{\max }$. Für den Fall eines Maximalabstandes $T_{i j}^{\max }$ setzen wir eine Folge von Abständen von $i$ nach $j$ voraus, so daß $T_{j i_{1}}^{m i n}+\ldots+T_{j_{\nu} i}^{\text {min }} \leq T_{i j}^{m a x}$. Im Netzplan werden die zeitlichen Minimalabstände im folgenden durch $T_{i j}^{m_{i j}}=b_{i j}$ und die zeitlichen Maximalabstände durch $T_{i j}^{\max }=-b_{j i}$ dargestellt. Durch die Maximalabstände enthält das Netzwerk Zyklen negativer Länge und das Finden einer zulässigen Lösung für das zugehörige Projektplanungsproblem ist $\mathcal{N} \mathcal{P}$ schwer.

In einer an Pritsker et al. 1969 angelehnten Notation stellt sich das Projektplanungsproblem RCPSP/max wir folgt dar:

$$
\begin{aligned}
& \min \sum_{t=E F T_{n}}^{T} x_{n t} t \\
& \text { u.d.N. } \sum_{t=E F T_{j}}^{L F T_{j}} x_{j t} \quad=1 \quad \forall j=1, \ldots, n \\
& \sum_{t=E F T_{i}}^{L F T_{i}} x_{i t}\left(t-D_{i}+b_{i, j}\right) \leq \sum_{t=E F T_{j}}^{L F T_{j}} x_{j t}\left(t-D_{j}\right) \quad \forall j=1, \ldots, n ; \\
& i \in P(j) \\
& \sum_{j=2}^{n-1} r_{j \kappa} \sum_{\tau=\max \left\{t, E F T_{j}\right\}}^{\min \left\{t+D_{j}-1, L F T_{j}\right\}} x_{j \tau} \leq R_{\kappa t} \quad \forall \kappa \in R ; t=1, \ldots, T \\
& x_{j t} \in\{0 ; 1\} \quad \forall j=1, \ldots, n ; \\
& t \in\left\{E F T_{j}, \ldots, L F T_{j}\right\}
\end{aligned}
$$

Der Projektendtermin wird durch die Zielfunktion (1) minimiert. Die Binärvariable $x_{j t}$ ist 1 , wenn der Vorgang $j$ zum Zeitpunkt $t$ beendet wird, ansonsten 0 . Gleichung (2) stellt sicher, daß jeder Vorgang nur innerhalb der zulässigen Zeit, die sich aus den Vorrangbeziehungen (3) ergeben, ausgeführt wird. Die Ressourcenbeschränkungen werden durch Gleichung (4) dargestellt. $R$ ist dabei die Menge aller erneuerbaren Ressourcen und $r_{j k}$ die Ressourcennachfrage von Vorgang $j$ nach Ressource $\kappa$, die in jeder Periode während der Ausführung dieselbe ist. $D_{j}$ steht für die Vorgangsdauer und $E F T_{j}\left(L F T_{j}\right.$ ) für den frühesten (spätesten) Endtermin des Vorgangs $j$, die sich aus einer MPM-Zeitplanung ergeben.

\section{Prioritätsregelverfahren für das RCPSP/max}

Bei Prioritätsregelverfahren wird zwischen seriellen und parallelen Verfahren unterschieden. Serielle Verfahren erzeugen aktive Pläne, parallele Verfahren unverzögerte. Während die Menge der aktiven Pläne stets einen optimalen Plan enthält, ist dies für die Menge der unverzögerten, die eine Teilmenge der aktiven 
Pläne ist, nicht gewährleistet. Trotzdem haben viele Untersuchungen gezeigt, daß die parallelen Verfahren im Mittel die besseren Ergebnisse liefern (s. z.B. Kolisch 1995). Aus diesem Grund wollen wir uns im folgenden auf die parallele Generierung einer Lösung beschränken.

Beim RCPSP/max treten im Gegensatz zum RCPSP Zyklen negativer Länge auf. Dies macht ein anderes Vorgehen notwendig. Alternative Vorgehen sind ein direktes Vorgehen, bei dem der gesamte zyklische Netzplan betrachtet wird oder ein Kontraktionsverfahren, bei dem die Zyklenstrukturen zunächst gesondert betrachtet werden, um den zyklischen Netzplan anschließend zu einem azyklischen Netzplan zu vereinfachen. Experimentielle Untersuchungen von Brinkmann und Neumann 1995 haben gezeigt, daß die Kontraktionsmethode normalerweise eher eine zulässige und bessere Lösung findet. Deshalb werden wir uns im folgenden auf die Kontraktion beschränken. Die Kontraktion der Zyklen basiert auf einem Theorem von Bartusch et al. 1988.

Theorem 1. Es gibt für das ressourcenbeschränkte Projektplanungsproblem $N$ genau dann eine Lösung, wenn für jede Zyklenstruktur $C$ von $N$ ein zulässiges Subschedule existiert.

\section{Algorithmus für das RCPSP/max \\ Stufe 1 Bestimme die Zyklenstrukturen \\ Stufe 2 Plane die Zyklenstrukturen getrennt \\ Stufe 3 Kontrahiere den Netzplan \\ Stufe 4 Plane den zyklenfreien Netzplan}

Die Bestimmung der Zyklenstrukturen kann mit einem Verfahren von Even 1979 durchgeführt werden, das eine Zeitkomplexität von $O(|E|)$ besitzt $(|E|$ ist die Anzahl der Pfeile in N).

Die Planung der Zyklenstrukturen (Stufe 2) enthält wiederum mehrere Teilschritte.

\section{Algorithmus für die Zyklenstrukturen}

Stufe 2.1 Erweitere Zyklenstruktur um eine künstliche Quelle und Senke

Stufe 2.2 Bestimme früheste und späteste Anfangszeitpunkte der Vorgänge durch Zeitplanung

Stufe 2.3 Führe eine ressourcenbeschränkte Planung der Zyklenstruktur mit Hilfe eines Prioritätsregelverfahrens durch

Die Erweiterung der Zyklenstruktur (Stufe 2.1) um eine Quelle $q$ und Senke $s$ ist erforderlich, um einen eindeutigen Startknoten für die spätere Planung zu erhalten, auf den sich die Startzeitpunkte beziehen. $J_{c}$ sei die Menge der Knoten $j \in V_{c}$ mit $b_{j k}<0$ für alle $\left\langle j, k>\in E_{c}\right.$ und $I_{c}$ sei die Menge der Knote $i \in V_{c}$ mit $b_{l i}<0$ für alle $\left\langle l, i>\in E_{c}\right.$. Füge für alle Vorgänge $i \in I_{c}$ einen Pfeil $<q, i>$ mit $b_{q i}=0$ und für alle $j \in J_{c}$ einen Pfeil $\left\langle j, s>\right.$ mit $b_{j s}=D_{j}$ ein.

Um den spätesten Anfangszeitpunkt zu bestimmen, muß zunächst der längste Weg $l_{j i}$ zwischen allen $j \in J_{c}$ und $i \in I_{c}$ bestimmt werden. Für den spätesten 
Anfangszeitpunkt der künstlichen Senke gilt

$$
L S T_{s}=\max _{i \in I_{c}}\left\{\max _{j \in J_{c}}\left\{-l_{j i}+D_{j}\right\}\right\} .
$$

Im Anschluß an eine MPM-Zeitplanung wird eine ressourcenbeschränkte Planung der Zyklenstrukturen mit einem parallelen Prioritätsregelverfahren durchgeführt. Dabei ist zu beachten, daß durch ein die Maximalabstände Vorgänge zu früh eingeplant können werden. In diesem Fall muß eine Rückplanung durchgeführt werden. Damit die Rückplanung möglichst vermieden wird, wird ein maximal spätester Anfangszeitpunkt $M S T_{i}^{c}$ für jeden Vorgang mitgeführt, der anfangs der späteste Anfangszeitpunkt ist. Dieser wird bei jeder Einplanung entsprechend geändert.

Nachdem für alle Zyklenstrukturen eine zulässige Lösung gefunden wurde (anderenfalls bricht das Verfahren hier ab) wird nun in Stufe 3 der zyklische zu einem azyklischen Netzplan kontrahiert. Zu diesem Zweck werden die jeweiligen Zyklenstrukturen zu einem neuen Knoten kontrahiert. Dabei müssen die Vorrangbeziehungen im Netzplan wie folgt geändert werden:

1. Ersetze alle Pfeile $\langle i, j\rangle$ mit $i \in V_{c} ; j \notin V_{c}$ durch einen Pfeil $<i_{c}, j>$ mit $b_{i_{c} j}=b_{i j}+S T_{i}^{c}$

2. Ersetze alle Pfeile $\left\langle k, i>\right.$ mit $i \in V_{c} ; k \notin V_{c}$ durch einen Pfeil $<k, i_{c}>\operatorname{mit} b_{k i_{c}}=\max \left\{0, b_{k i}-S T_{i}^{c}\right\}$

3. Ersetze alle Pfeile $\langle j, l\rangle$ mit $j \in V_{c_{1}} ; l \in V_{c_{2}}$ durch einen Pfeil

$$
<j_{C_{1}}, l_{c_{2}}>\operatorname{mit} b_{l_{c_{1}} j_{c_{2}}}=\max \left\{0, b_{j l}+S T_{j}^{c_{1}}-S T_{l}^{c_{2}}\right\}
$$

Der Ressourcenbedarf der Zyklenstruktur wird im Gegensatz zu Brinkmann und Neumann 1995 jedoch nicht mit den jeweiligen Maxima der Ressourceninanspruchnahme angenommen, sondern mit den tatsächlichen Werten, die sich im Zeitverlauf ändern können. Dieses zeitliche Schwanken der Ressourcennachfrage der kontrahierten Zyklenstrukturen ermöglicht bessere Lösungen als die Maximumbildung

In Stufe 4 wird ein paralleles Prioritätsregelverfahren für das kontrahierte azyklischen Projektplanungsproblem durchgeführt. Dabei muß die zeitliche schwankende Ressourcennachfrage der kontrahierten Zyklenstruktur-Knoten beachtet werden.

Die Verbesserung gegenüber dem Verfahren in Zhan 1991 besteht darin, daß eine Kontraktion des Netzplans durchgeführt wird. Dadurch braucht eine MPMZeitplanung nur für den kontrahierten und nicht für den zyklischen Netzplan durchgeführt werden. Außerdem fallen bei der Planung des gesamten (kontrahierten) Projektplanungsproblems im Gegensatz zu Zhan keine zeitintensiven Rückplanungen mehr an.

\section{Prioritätsregeln und Sampling}

Für das RCPSP/max sind nach Zhan 1991 die folgenden Prioritätsregeln am besten geeignet: Latest Start Time (LST), Latest Finish Time (LFT), Resource 
Scheduling Method (RSM), Longest Path Following (LPF), Most Total Successors (MTS), Least Float per Successor (LFS).

Neben der Untersuchung einzelner Prioritätsregeln hat Zhan auch die Lösung mit Hilfe eines Sampling-Verfahrens untersucht, das sogenannte "kombinierte" Prioritätsregel-Verfahren. Dabei hat er bis zu vier Prioritätsregeln nacheinander angewendet und anschließend die beste Lösung ausgewählt.

Für das RCPSP sind nach Kolisch 1995 folgende weitere Prioritätsregeln sehr gut geeignet: Worst Case Slack (WCS), Improved RSM (IRSM), Minimum Slack Time (MSLK). Kolisch untersuchte neben den einzelnen Prioritätsregeln ferner das Regret Biased Random Sampling, bei dem eine bestimmte Anzahl von Lösungen mit derselben Prioritätsregel erzeugt werden, um dann die beste auszuwählen. Hierbei handelt es sich um eine Auswahl der Vorgänge beim scheduling, wobei dieser Zufall jedoch durch Prioirtätsregeln und ein Regret-Maß beeinflußt werden. Die Verwendung diese Random Sampling zusammen mit den Regeln LFT und WCS haben sich als erfolgreich herausgestellt.

\section{Die Testprobleme}

Um die Ergebnisse von Zhan und Kolisch zu überprüfen, benutzen wir Testprobleme, die mit ProGen/max erzeugt wurden. In unserem Fall handelt es sich um Probleme, die $n=100$ Vorgänge besitzen und durch folgende Kennzahlen charakterisiert werden: Restrictiveness $R T$, Ressource Faktor $R F$, Resource Strength $R S$ sowie die Größe der Zyklenstruktur $n_{c}$ und die Anzahl der Zyklenstrukturen $C S$ (vgl. Schwindt 1995).

Anstatt der bei Kolisch 1995 verwendeten Netzwerkkomplexität $N C$ verwenden wir die Restrictiveness nach Thesen 1977, die besagt, wieviele verschiedene zulässige Schedulings eines Netzplanes ohne Ressourcenbeschränkung es gibt. Die Ressourcenkennzahlen $R F$ und $R S$ übernehmen wir von Kolisch. Für die Charakterisierung der Zyklenstrukturen verwenden wir die relative Anzahl von Maximalabständen bezogen auf die Anzahl der $m$ der Pfeile sowie die relative Anzahl von Zyklenstrukturen $C S$ bezogen auf die maximal mögliche Anzahl von $n / 2$. Es werden wie bei Kolisch nur redundanzfreie Projektpläne erzeugt. Die übrigen Steuerparameter (vgl. Schwindt 1995) wurden fest vorgegeben. Auf eine umfangreiche Aufzählung soll hier verzichtet werden.

Für die ersten 3 Kennzahlen wählen wir folgende Parameter:

$$
\begin{array}{lll}
N C & =\{1,5 ; 1,8 ; 2,1\} & \\
R F & =\{0,25 ; 0,5 ; 0,75 ; 1\} & \\
R S & =\{0,2 ; 0,5 ; 0,7\} & \\
M T L & =\{0.1 ; 0.25\} & \operatorname{Max}(\mathrm{MTL})=\mathrm{MTL}+0.1 ; \operatorname{Min}(\mathrm{MTL})=\mathrm{MTL} \\
C S & =\{0.1 ; 0.25\} & \operatorname{Max}(\mathrm{CS})=\mathrm{CS}+0.1 ; \operatorname{Min}(\mathrm{CS})=\mathrm{CS}
\end{array}
$$

Für jede mögliche Parameterkombination werden 10 Probleme erzeugt, so daß sich insgesamt $2^{5} * 10=320$ Probleme ergeben. 


\section{Testergebnisse}

In der nachfolgenden Tabelle sind die Ergebnisse für das obige Testset aufgeführt. Es sind die mittleren Abweichungen vom jeweils besten Wert und der mittlere Rang angegeben. Es ist erkennbar, daß alle getesteten Regeln im Mittel einen besseren Wert liefern als der Zufall.

\begin{tabular}{|c|c|c|c|c|c|c|c|c|}
\hline & MSLK / LST & WCS & MTS & LFS & IRSM & RSM & LPF & Random \\
\hline rel. Abw. & $3,76 \%$ & $3.92 \%$ & $3,93 \%$ & $4,13 \%$ & $4,52 \%$ & $4,92 \%$ & $5,03 \%$ & $6,38 \%$ \\
\hline Rang & 2,36 & 3,48 & 2,77 & 3,04 & 3,16 & 3,8 & 3,89 & 6,00 \\
\hline
\end{tabular}

Da wir ein paralleles Generierungschema gewählt haben, haben die Regeln LST und MSLK erwartungsgemäß dieselbe Lösung geliefert. Es läßt sich festhalten, daß wir eine etwas anderes Ergebnis erzielen als Kolisch 1995, bei dem die Reihenfolge WCS, IRMS, LFT, RSM bzw. LST, LFT, MTS lautet. Auch die von Zhan ermittelte Reihenfolge sieht etwas anders aus: LFT, RSM, LST, LPF, MTS, LFS. Ob diese Unterschiede nur auf die Zyklenstrukturen zurchzuführen sind, oder auch durch die Erzeugung der Probleme mit Hilfe der Restritivenes $R T$ anstatt der Netzwerkkomplexität $N C$ bzw. der übrigen Parameter von ProGen/max entstehen bedarf weitere Untersuchungen. Ferner wird geklärt werden, ob die von Kolisch präferierte Random-Sampling-Methode oder das von Zhan empfohlene Sampling mit verschiedenen ausgesuchten Prioritätsregeln bessere Ergebnisse liefert. Um die Vergleichbarkeit zu gewährleisten ist die Anzahl der Samplings für das Random-Sampling auf dieselbe Anzahl der kombinierten Prioritätsregeln festzulegen

\section{References}

1. Bartusch, M., Möhring, R.H., Radermacher, F.J. (1988): Scheduling project networks with resource constraints and time windows; Annals of Operations Research $16,201-240$

2. Kolisch, R. (1995): Project Scheduling Under Resource Constraints - Efficient Heuristics for Several Problem Classes; Physica, Heidelberg

3. Pritsker, A.A.B., Watters, W.D., Wolfe, P.M. (1969): Multiproject scheduling with limited resources: A zero-one programming approach; Management Science 16, 93-107

4. Schwindt, C. (1995): ProGen/max: A new problem generator for different resourceconstrained project scheduling problems with minimal and maximal time lags; Report WIOR-449, Institut für Wirtschaftstheorie und Operations Research, Universität Karlsruhe

5. Thesen, A. (1977): Measures of restrictiveness of project networks; Networks 7, $193-202$

6. Zhan, J. (1991): Heuristische Ressourcenplanung in MPM-Netzplänen mit beschränkter Kapazität; Dissertation, Universität Karlsruhe 